\title{
A Novel Protocol Permitting the Use of Frozen Cell Cultures on Low Earth Orbit
}

\author{
L. S. Kidder ${ }^{1}$, L. Zea ${ }^{2}$, SM Countryman², L. S. Stodieck², B. E. Hammer ${ }^{1, *}$
}

${ }^{1}$ Department of Radiology, Center for Magnetic Resonance Research, University of Minnesota, Minneapolis, MN; '2BioServe Space Technologies, University of Colorado, Boulder, CO

Abstract

Cell culture on orbit is complicated by numerous operational constraints, including g-loads on the ascent, vibrations, transit time to International Space Station, and delays in experiment initiation. Cryopreserving cells before launch would negate these factors. However, defrosting these cells is problematic, since the traditional method of employing a water bath is not possible. We here describe a unique apparatus designed to accomplish this in a microgravitational environment. This apparatus resulted in rapid defrost of cryopreserved cell cultures and allowed successful tissue culture operations on the station for periods of up to 21 days.

Keywords

Cryopreserved cells • Defrost frozen cultures on orbit • Cell culture on ISS

\section{INTRODUCTION}

The use of mammalian cells in Low Earth Orbit to study the effects of microgravity on cellular biology is fraught with several possible complicating factors. Cultures of adherent cells are necessarily initiated in the laboratory; they then must be transported to orbit. Doing so often involves delays of hours to days. In addition, cultures are subject to high g loads, accelerations, and intense vibrations on the ascent (Kacena et al., 2003). Once on orbit, it normally takes several days before studies can commence, due to the time required for spacecraft docking to the International Space Station (ISS), unloading the transport vehicle, and processing the delivered material.

Freezing cells before launch provide a way to mitigate these issues. Cells may be cultured in the laboratory following standard protocols and frozen at a controlled rate in media supplemented with $10 \% \mathrm{v} / \mathrm{v}$ dimethyl sulfoxide (DMSO) (Freshney, 1987). When cryopreserved, cultures are isolated from the forces the spacecraft experiences during launch. Since they are effectively in stasis, the initiation of experimental protocols can wait for a time congruent with crew duties. However, the rapid and consistent defrosting of cell cultures is challenging in a microgravitational environment. Frozen cells on Earth (typically in a cryovial) may be rapidly thawed by agitation in a $37^{\circ} \mathrm{C}$ water bath. However, the use of an open water bath is not possible on the ISS. We here describe the development of a contained system that allows the rapid thawing of adherent cell cultures.

\section{COMPARATIVE DEFROST METHODS}

In developing an on-orbit defrost system, we examined alternative methods to rapidly defrost an enclosed large volume of ice. The key to minimizing cell death during the thawing of frozen cells is to ensure rapid heat transfer into the ice mass leading to rapid thawing (Mazur, 1984). The specific heat capacity of the cell culture and material surrounding the frozen cell culture, that is, the BioCell cell culture plate (BioServe Space Technologies; Boulder, CO) (Figure 1), determines the rate at which the cell culture temperature rises from a solid to liquid to the phase transition. The specific heat capacity is defined as:

$$
\mathrm{C}=\Delta \mathrm{Q} / \mathrm{m} \Delta \mathrm{T} \cdot \mathrm{kJ}-\mathrm{kg}^{-1}-\mathrm{K}^{-1}
$$

where $\Delta Q(\mathrm{~J})$ is the heat input, $\mathrm{m}$ is the mass of the BioCell + frozen media, and $\Delta \mathrm{T}$ is the temperature rise. $\mathrm{C}$ for liquid water $=4.2 \mathrm{~J}-\mathrm{kg}^{-1}-\mathrm{K}^{-1}$, for ice $2.1 \mathrm{~kJ}-\mathrm{kg}^{-1}-\mathrm{K}^{-1}$ with a heat of fusion/

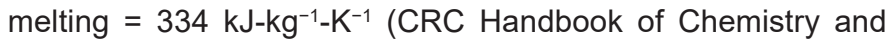
Physics, 1981). We initially attempted to use aluminum blocks to 

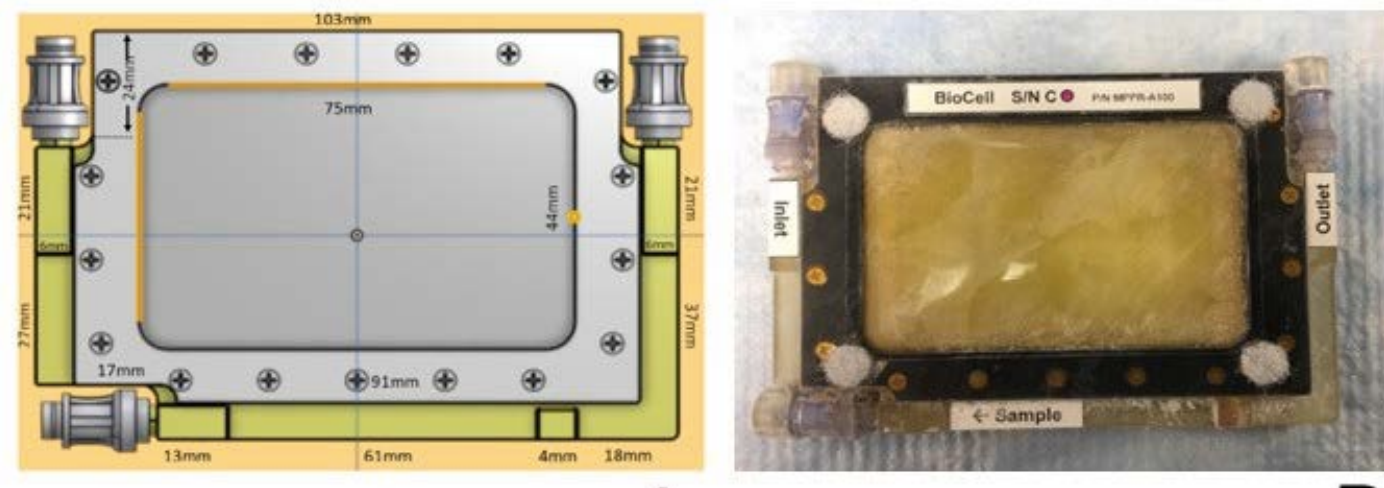

A

Figure 1. (A) Engineering drawing of BioCell and (B) BioCell with frozen cell culture/media. The needle-less Luer connectors allow the exchange of media.

warm the BioCell to avoid the complication of handling water bags on-orbit (Figure 2). However, since the heat capacity of

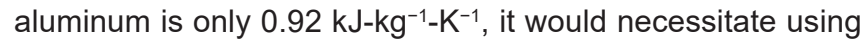
aluminum blocks with a mass of several $\mathrm{kg}$ to be capable of holding the initial temperature of $43^{\circ} \mathrm{C}$ necessary to thaw a frozen BioCell containing $30 \mathrm{ml}$ of cryopreservation media. Aluminum has high thermal conductivity $(236 \mathrm{w} / \mathrm{m}-\mathrm{K})$ (CRC Handbook of Chemistry and Physics, 1981) and can rapidly transfer heat to a frozen BioCell. However, in the environment found on the ISS, the aluminum block will lose much of its heat to the atmosphere before an astronaut can place the block in contact with a BioCell. Furthermore, once in contact with a BioCell, the surface immediately adjacent rapidly cools, effectively limiting the rate of heat transfer. An additional complication arises in that there is a nonperfect contact between the BioCell surface and the aluminum block since the flat plane of the block must meet the convex surface of the frozen BioCell (Figure 2). These factors directed us toward designing a device to hold an aqueous medium to rapidly warm the frozen cell cultures. In a liquid, all surfaces of the BioCell are in intimate contact with the heat transfer medium (i.e., water); immersion in a liquid allows mixing the solution so that the BioCell will be in intimate contact with the average bath temperature, not with a cooler boundary/ surface.

\section{MATERIALS AND METHODS}

Fibroblastic osteoprogenitor cells (MC3T3-E1; subclone 4) (ATCC; Manassas, VA) were cultured on modified culture plates developed by BioServe Space Technologies (Boulder, CO) (Figure 1). These culture vessels (BioCells) are constructed of an aluminum frame surrounding a $2 \mathrm{~mm}$ thick tissue-culture treated polystyrene (Corning; Corning, NY) on one side and

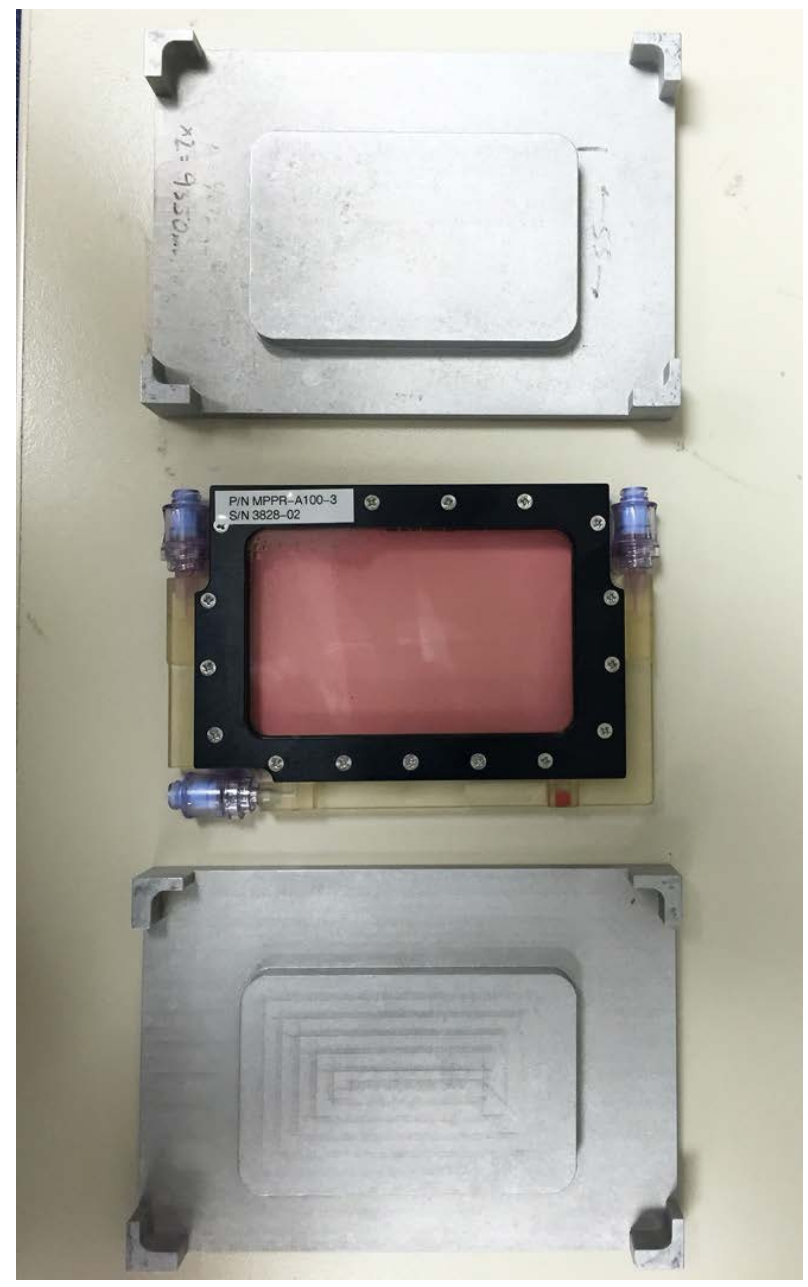

Figure 2. Prototype aluminum block designed to defrost frozen BioCells on orbit. Both sides of this block were heated to $43^{\circ} \mathrm{C}$ and positioned on the frozen BioCell. It was ultimately determined that aluminum was not effective in rapidly defrosting frozen cultures. 

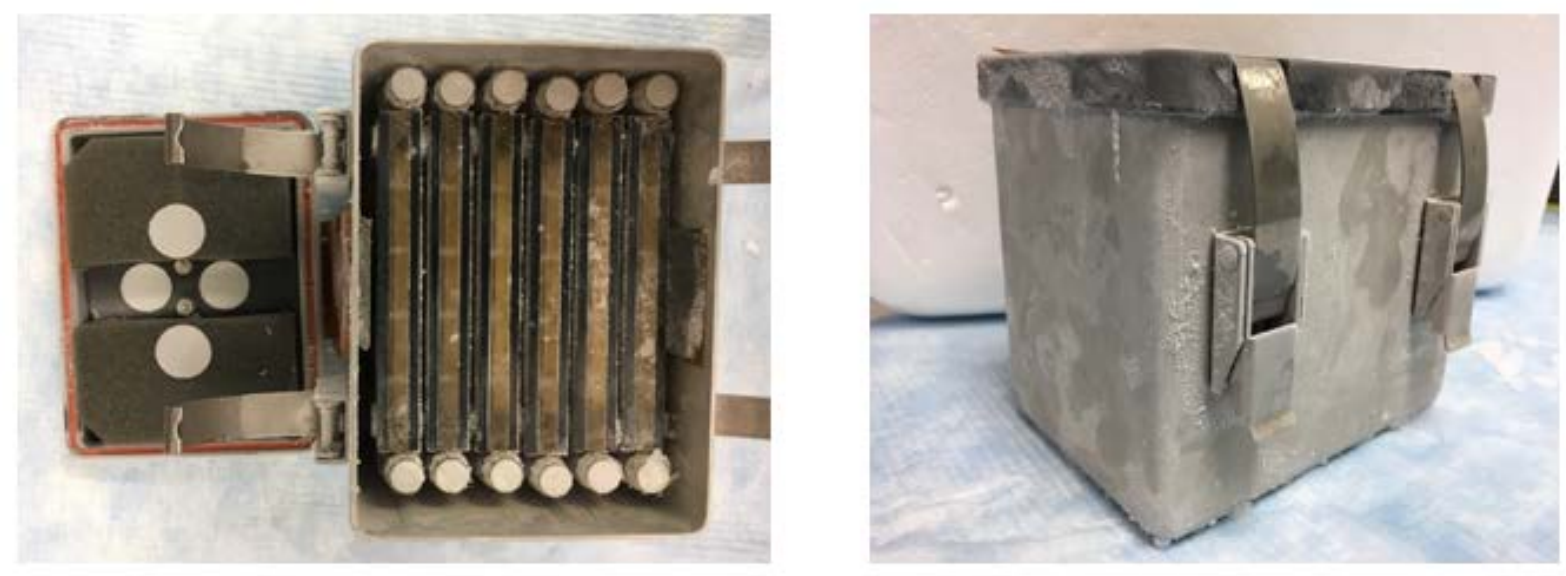

Figure 3. Metal enclosure housing BioCells (PHAB). Note the vents at the top of the enclosure allowing gas exchange while it is maintained in the $37^{\circ} \mathrm{C}, 5 \% \mathrm{CO}_{2}$ incubator.

a $0.1 \mathrm{~mm}$ thick gas permeable FEP Teflon membrane on the other side; each has a surface area of $50 \mathrm{~cm}^{2}$ containing a volume of $30 \mathrm{ml}$. Ports in the frame allow for cell seeding as well as the introduction and withdraw of culture media.

\section{Freezing Protocol}

Cryopreserved cells may be stored for an extended period in liquid nitrogen $\left(77^{\circ} \mathrm{K} ;-196^{\circ} \mathrm{C}\right)$. During cryopreservation, the rate of cooling is crucial to maximizing cell survival upon defrosting. Cellular injury during freezing is typically associated with osmotic differences across the cell membrane and the formation of intracellular ice. If cooled at a slow controlled rate $\left(-1^{\circ} \mathrm{C} / \mathrm{min}\right)$, water migrates out of the intracellular space through exosmosis so that the cell dehydrates and does not freeze intracellularly. If cooling is too rapid, the interior of the cell achieves thermal equilibrium with the extracellular space by freezing (Seth, 2012). For this project, cells were defrosted and cultured in T-175 or T-225 flasks per standard protocols (Freshney, 1987). Cells were then allowed to proliferate to approximately $80 \%$ confluence and passed to BioCells at either $10,000 / \mathrm{cm}^{2}$ or $50,000 / \mathrm{cm}^{2}$. After $24 \mathrm{~h}$ in culture, media was replaced with cryopreservation media, composed of alpha-MEM (Gibco/ ThermoFisher; Waltham, MA) + 10\% Fetal Bovine Serum (v/v) (Sigma-Aldrich; St Louis, MO) $+1 \%$ penicillin/streptomycin (10k U/ml/10k U/ml) (Gibco/ThermoFisher; Waltham, MA) and supplemented with 10\% DMSO (v/v) (Sigma-Aldrich; St Louis, MO). Cultures were then frozen at a controlled rate of $-1^{\circ} \mathrm{C} / \mathrm{min}$ (Seth, 2012) (Cryomed Controlled-Rate Freezer; Thermo-Fisher Scientific; Waltham, MA) and stored at $-80^{\circ} \mathrm{C}$ in a metal enclosure (PHAB; BioServe Space Technologies; Boulder, CO) (Figure 3). SpaceX CRS-9 (launched from Kennedy Space Center, FL July 18, 2016) transported these frozen cell cultures to the ISS in a POLAR freezer $\left(<-80^{\circ} \mathrm{C}\right)$ (berthed July 20, 2016). Once on orbit, frozen BioCells were stored in the Minus Eighty-Degree Laboratory Freezer for ISS (MELFI) at $-95^{\circ} \mathrm{C}$ until the start of the experiment. The anticipated experiment start was delayed several months due to the failure of an essential incubator component. A replacement part and fresh frozen culture media were later delivered to ISS (February 2017), and the experiment was initiated on May 2, 2017.

\section{BioServe Thaw System}

Rapid thawing is necessary to minimize the formation of ice crystals and osmotic stressors that can damage cryopreserved cells (Seth, 2012; Ragoonanan et al., 2013). To accomplish this, we have developed a defrost apparatus that consists of two connected ethylene-vinyl acetate bags ( $0.35 \mathrm{~mm}$ thick) closed with clips (Figure 4). One bag is preloaded with water acting as a reservoir, the other, smaller, bag is empty. A clip is situated over the isthmus between them, to isolate them. A $60 \mathrm{ml}$ syringe connects a port connected to the empty bag, allowing for the complete withdrawal of air, while another $60 \mathrm{ml}$ syringe transfers the remaining water from the thawing chamber back to the reservoir.

To thaw frozen BioCells, the system is preheated to $43^{\circ} \mathrm{C}$ in an oven aboard the ISS. This temperature was chosen based on trials with frozen (cell free) BioCells (data not shown). It was determined that since the relatively small volume of water used by this system cools fairly rapidly it is necessary to initiate the process with a slightly higher temperature than the standard of $37^{\circ} \mathrm{C}$ to quickly complete defrost. This is demonstrated in Figure 5, which shows that, in the laboratory, a starting temperature of $43^{\circ} \mathrm{C}$ defrosts a BioCell within 2 min from a temperature of $-80^{\circ} \mathrm{C}$. The BioCell was removed from MELFI and placed in the thawing chamber bag. This bag was then sealed by folding the opening and clamping shut 


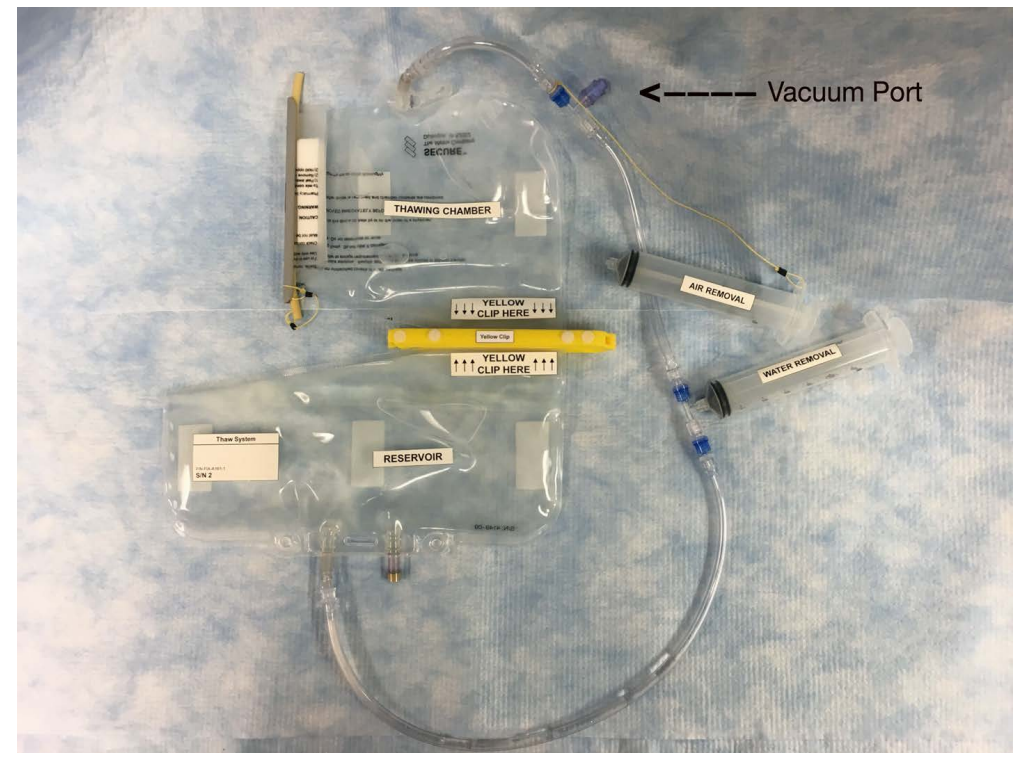

Figure 4. BioCell thaw system. The bag labeled "Reservoir" contains water heated to $43^{\circ} \mathrm{C}$. The frozen BioCell is placed in the bag labeled "Thawing Chamber" which is then sealed. Air is evacuated, and a large clip is opened between the two bags, and warm water pushed in. The thawing bag is again sealed. With rocking/kneading, the BioCell is defrosted within 2-3 min. The clip between the two bags is then opened, and water is pushed back into the reservoir bag.

with a clip. Air was evacuated from this bag by a syringe attached to the vacuum port. The large yellow clip depicted in Figure 4 was opened, and warm water was pushed from the reservoir into the thawing chamber. This clip was then reclosed, and the thawing chamber was gently massaged and lightly shaken (while holding the BioCell in place at a corner) to ensure consistent mixing of the warmed water surrounding the BioCell. After the BioCell had defrosted, the large clip was opened, and the remaining water pushed back to the reservoir from the thawing chamber which was again sealed by the large clip. The remaining water in the thawing chamber was removed through the syringe labeled "water removal" in Figure 4. Water sucked into this syringe was pushed into the reservoir using an inline one-way check valve. After all water had been evacuated from the thawing chamber bag, the clip which closed it was removed and the BioCell removed. This process took 2-3 min. The defrosted culture media + DMSO was then exchanged with fresh media, and the culture was placed in a $37^{\circ} \mathrm{C}, 5 \% \mathrm{CO}_{2}$ incubator (i.e., Space Automated Bioprocessing Laboratory designed and built by BioServe Space Technologies; Boulder, CO). The defrost system was then returned to the $43^{\circ} \mathrm{C}$ oven and another employed for the next frozen culture removed from the MELFI. Twenty-four cultures were defrosted and preserved on the ISS while 24 cultures were employed as asynchronous ground controls. Cell survival post-defrost in situ was successful, as shown in Figure 6 and Table 1.
Table 1. Quality and quantity of RNA recovered from flight cultures as determined by UV spectroscopy.

\begin{tabular}{ccc}
\hline ID & $\sim$ Quality $($ A260 $\lambda /$ A280 $\lambda$ ) & Total quantity $(\mu \mathbf{g})$ \\
\hline SN1 - D & 2.13 & 21.04 \\
SN1 - E & 2.05 & 12.76 \\
SN1 - F & 2.10 & 19.43 \\
SN2 - D & 2.06 & 4.81 \\
SN2 - E & 2.10 & 8.86 \\
SN2 - F & - & 0.00 \\
SN3 - D & - & 0.00 \\
SN3 - E & 2.10 & 18.22 \\
SN3 - F & 2.10 & 8.25 \\
SN4 - D & 2.00 & 2.24 \\
SN4 - E & 2.12 & 39.41 \\
SN4 - F & 2.14 & 39.49 \\
\hline
\end{tabular}

\section{Key}

SN1: Confluent; standard media. 13d culture.

SN2: Proliferating; standard media. 13d culture.

SN3: Confluent; differentiation media. 20d culture.

SN4: Proliferating; differentiation media. 20d culture.

Cellular nucleic acids were stabilized on-orbit with RNAprotect (Qiagen). Upon return, RNA was isolated with a guanidine-isothiocyanate-containing lysis buffer and purified by passing through a silica membrane that specifically binds RNA (Rnasy; Qiagen). The ratio of optical absorbance at $260 \mathrm{I}$ and $280 \mathrm{I}$ wavelengths is an indicator of RNA integrity and purity. Values above 1.8 are considered ideal (Glasel, 1995). The figure key refers to specific experimental treatments. 


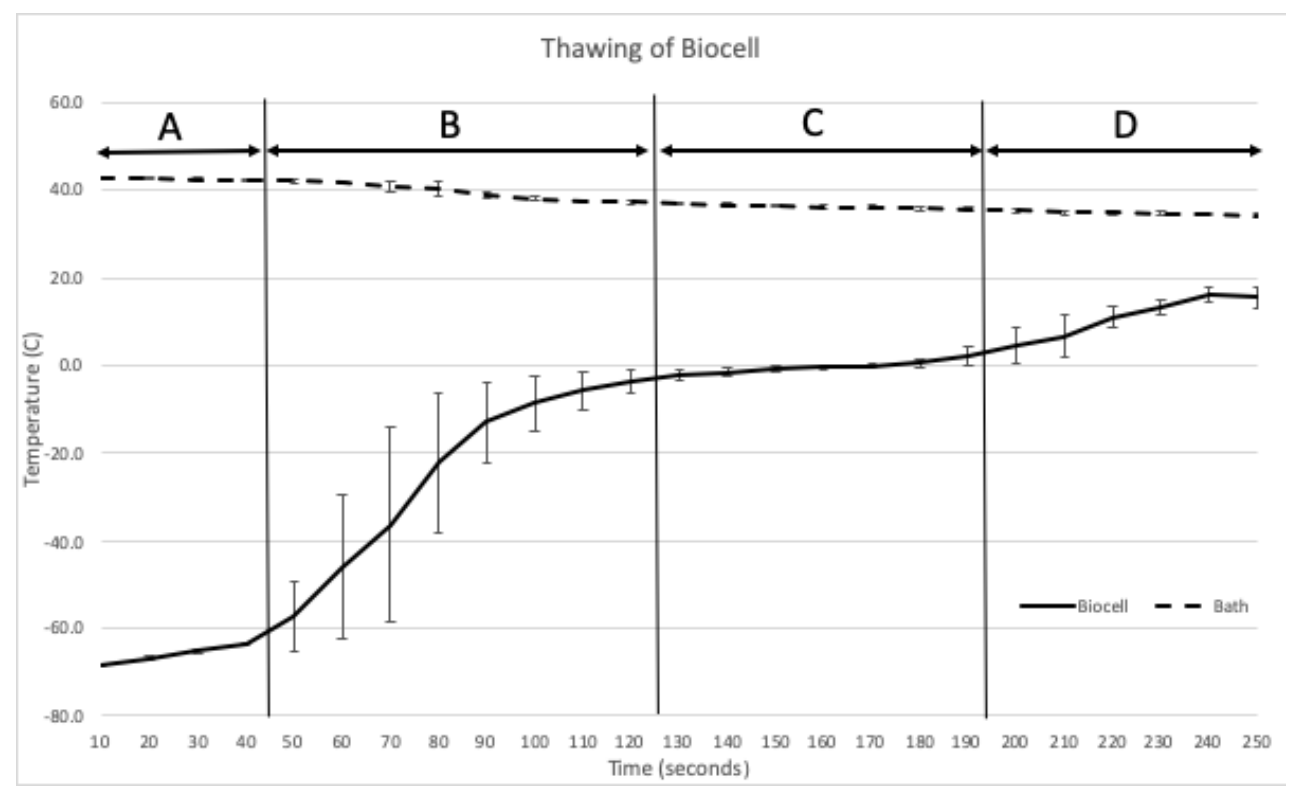

Figure 5. Time course for thawing of BioCell from $-80^{\circ} \mathrm{C} ; n=4$. The internal temperature of the BioCell was determined using a resistance temperature detector (RTD) (Omega HSRTD-3-100-A-80-E, Omega Engineering, Inc., Norwalk, CT) interfaced to LabVIEW 2018 (National Instruments, Austin, TX) through a customized virtual instrument and sampled at $1 \mathrm{~s}$ increments. (A) BioCell exposed to air at $20^{\circ} \mathrm{C}$.

(B) BioCell submerged in $1,000 \mathrm{ml}$ water bath $(23 \mathrm{~cm} \times 28 \mathrm{~cm})$ initially at $43^{\circ} \mathrm{C}$. Water depth is $19 \mathrm{~mm}$ before submerging BioCell. The standard deviation is higher because the physical location of the RTD in a frozen BioCell is variable and within a few millimeters of the BioCell membrane. During the thawing process, the BioCell is mechanically agitated to ensure the maximum convection/heat transfer occurs between the BioCell and bath. When an RTD is close to the BioCell membrane, which is in contact with the water bath, it will warm faster than an RTD further from the membrane. (C) Frozen media changing to liquid media. (D) Cell media in liquid state approaches bath temperature. Error bars are displayed every $10 \mathrm{~s}$ for the BioCell and bath but are not visible due to when the standard error is small, for example, at $150 \mathrm{~s}$ bath temperature is $37.2^{\circ} \pm 0.3^{\circ} \mathrm{C}$ and BioCell temperature is $-3.6^{\circ} \pm 2.6^{\circ} \mathrm{C}$.

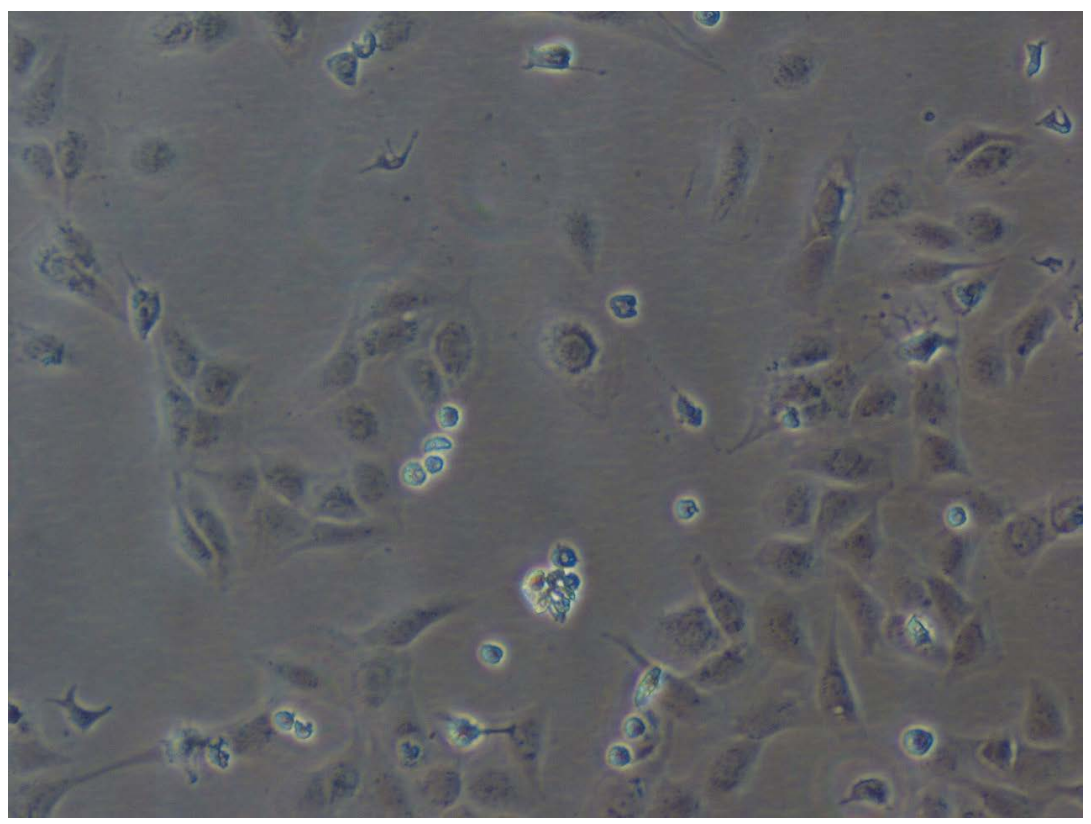

Figure 6. Photomicrograph of МС3Т3 osteoblastic cells post-defrost in situ (100x). Cells survived cryopreservation and proliferated normally. 


\section{GROUND-BASED VERIFICATION OF THAWING PROTOCOL}

When a BioCell is removed from a $-80^{\circ} \mathrm{C}$ environment and placed in the air at $20^{\circ} \mathrm{C}$ ambient air temperature, it warms at a rate of $9.9^{\circ} \pm 0.8^{\circ} \mathrm{C} / \mathrm{min}(N=4)$ in a $1-\mathrm{g}$ environment (Figure $5)$. Warming is likely slower in a microgravity environment because of the lack of buoyancy-driven air convection adjacent to the BioCell. In our laboratory, the BioCell was completely thawed within 2 min of submersion in the thawing system $(n=4)$ (Figure 5). Our results are comparable to observations aboard the ISS. A BioCell enters the thawing system after removal from MELFI. Video recordings of astronauts thawing a BioCell on the ISS demonstrated complete thawing the cell culture in $2 \mathrm{~min}$.

\section{SUMMARY}

We here describe a novel apparatus designed to consistently defrost cryopreserved cell cultures in the ISS environment. The system is closed and is easily deployed, thereby protecting the ISS crew from exposure to free-floating fluids. It can potentially be employed to defrost a variety of mammalian cell cultures and other biological materials.

\section{ACKNOWLEDGMENTS}

Research was supported by NIH/NIBIB UH2/UH3 EB013647 and CASIS (Center for the Advancement of Science in Space, Inc.) cooperative agreement with NASA (NASANNH11CD70A).

\section{AUTHOR DISCLOSURE STATEMENT}

No competing financial interests exist for any of the authors.

\section{REFERENCES}

CRC Handbook of Chemistry and Physics (1981) 62nd edn. Boca Raton, Florida, USA: CRC Press.

Freshney R (1987) Culture of Animal Cells: A Manual of Basic Technique, New York: Alan R. Liss, pp 220.

Glasel JA (1995) Validity of nucleic acid purities monitored by 260nm/280nm absorbance ratios. Biotechniques 18(1), 62-63.

Kacena MA, Todd P, Landis WJ (2003) Osteoblasts subjected to spaceflight and simulated Space Shuttle launch conditions. Vitro Cell Dev Biol - Animal 39, 454-459.
Mazur P (1984) Freezing of living cells: mechanisms and implications. American Journal of Physiology 247, C125-42.

Ragoonanan V, Less R, Aksan A (2013) Response of the cell membrane-cytoskeleton complex to osmotic and freeze/thaw stresses. Part 2: The link between the state of the membrane-cytoskeleton complex and the cellular damage. Cryobiology 66(2), 96-104.

Seth G (2012) Freezing mammalian cells for production of biopharmaceuticals. Methods 56(3), 424-431. 